\title{
Nonlinear Instability for a Volume-Filling Chemotaxis Model with Logistic Growth
}

\author{
Haiyan Gao ${ }^{1,2}$ and Shengmao $\mathrm{Fu}^{3}$ \\ ${ }^{1}$ College of Mathematics and Statistics, Northwest Normal University, Lanzhou 730070, China \\ ${ }^{2}$ School of Statistics, Lanzhou University of Finance and Economics, Lanzhou 730020, China \\ ${ }^{3}$ School of Mathematics and Computing Science, Guilin University of Electronic Technology, Guilin 541004, China
}

Correspondence should be addressed to Haiyan Gao; gaohy_54@sina.com

Received 2 April 2014; Accepted 8 June 2014; Published 3 July 2014

Academic Editor: Yongli Song

Copyright (C) 2014 H. Gao and S. Fu. This is an open access article distributed under the Creative Commons Attribution License, which permits unrestricted use, distribution, and reproduction in any medium, provided the original work is properly cited.

\begin{abstract}
This paper deals with a Neumann boundary value problem for a volume-filling chemotaxis model with logistic growth in a $d$ dimensional box $\mathbb{T}^{d}=(0, \pi)^{d}(d=1,2,3)$. It is proved that given any general perturbation of magnitude $\delta$, its nonlinear evolution is dominated by the corresponding linear dynamics along a finite number of fixed fastest growing modes, over a time period of the order $\ln (1 / \delta)$. Each initial perturbation certainly can behave drastically different from another, which gives rise to the richness of patterns.
\end{abstract}

\section{Introduction}

An important variant of the chemotaxis model was initially proposed by Painter and Hillen in [1] to model the volumefilling effect. In the volume-filling effect, it is assumed that cells have a certain finite (nonzero) volume and that the occupation of an area limits other cells from penetrating it. A simple version of the volume-filling chemotaxis model (VF) is the following:

$$
\begin{aligned}
& U_{t}=\nabla\left(d_{1} \nabla U-\chi U(1-U) \nabla V\right), \\
& V_{t}=d_{2} \nabla^{2} V+\alpha U-\beta V,
\end{aligned}
$$

where $d_{1}, d_{2}, \chi, \alpha$, and $\beta$ are given positive constants. $U(x, t)$ is the cell density and $V(x, t)$ denotes the density of the external chemical substance which is secreted by the cells themselves. $d_{1}$ and $d_{2}$ denote the cell and chemical diffusion coefficients, respectively. $\chi$ is called chemosensitivity. The term $U(1-U) \nabla V$ denotes the chemotactic flux under a volume constraint 1 (called crowding capacity), meaning that the chemotactic movement will be inhibited at the'aggregation location where the cell density reaches 1 .

In recent years, the chemotaxis models with volume-filling effect have been studied extensively. Hillen and Painter [2] firstly proved the global existence of solutions. Numerical simulations in one and two dimensions show interesting phenomena of pattern formation and formation of stable aggregates. Wrzosek [3] showed the existence of a compact global attractor in the space $W^{1, p}\left(\Omega, \mathbb{R}^{2}\right), p>n, \Omega \subset \mathbb{R}^{n}$ for some cases. In [4], the structure of the attractor can be understood using Lyapunov functions. Stationary solutions which are inhomogeneous in space were investigated for a given range of parameters. In [1], a numerical exploration was conducted to determine the longtime patterning behaviour, revealing formation of multiple plateau type patterns which undergo a coarsening process with increasingly long transient times. Potapov and Hillen [5] investigated the metastability of steady states. The underlying bifurcation diagram was identified, revealing that the unstable eigenvalues are exponentially small. The plateau interactions were studied using asymptotic methods. In [6], it was obtained that a priori estimates for the classical chemotaxis model of Patlak, Keller, and Segel when a nonlinear diffusion or a nonlinear chemosensitivity was considered accounting for the finite size of the cells and how entropy estimates give natural conditions on the nonlinearities implying the absence of blow-up for the solutions were showed. Burger et al. [7] discussed 
the effects of linear and nonlinear diffusion in the large time asymptotic behavior of the Keller-Segel model of chemotaxis with volume-filling effect. Moreover, the global existence of solutions and nontrivial steady states were also studied. Wang and Hillen [8] established the global existence of classical solutions to a generalized chemotaxis model, which includes the volume-filling effect expressed through a nonlinear squeezing probability. Necessary and sufficient conditions for spatial pattern formation were given and the underlying bifurcations were analyzed. In [9], the stationary solutions of the volume-filling chemotaxis model without a growth term were obtained by Jiang and Zhang.

Moreover, Wrzosek [10] considered various assumptions on nonlinear diffusion and chemotactic sensitivity function which lead to the existence of global in time solutions, thus preventing blow-up. In [11], Winkler proved that if certain conditions were fulfilled, then there were solutions that blow up in either finite or infinite time. In particular, in the framework of chemotaxis models incorporating a volume-filling effect in the sense of Painter and Hillen [1], his results indicated how strongly the cellular movement must be inhibited at large cell densities in order to rule out chemotactic collapse. Winkler and Djie [12] discussed boundedness and finite-time collapse for a chemotaxis system with volume-filling effect. Wang et al. [13] proved that for a wide range of nonlinear diffusion operators, including singular and degenerate ones, if the taxis force was strong enough with respect to diffusion and the initial data were chosen properly, then there was a classical solution which reaches the threshold at the maximal time of its existence; no matter whether the latter was finite or infinite. Zhang and Zheng [14] obtained the crucial uniform boundedness of the solution for a quasilinear nonuniform parabolic system modelling chemotaxis with volume-filling effect and the results on convergence to equilibrium and the decay rate using a suitable nonsmooth Simon-Łojasiewicz approach. In [15], the uniform boundedness, global in time existence and uniqueness of classical solution, were proved. With the help of a suitable nonsmooth Simon-Łojasiewicz approach, the results on convergence of the solution to equilibrium and the convergence rate were obtained. Li and Zhang [16] classified the existence or nonexistence of steady state solutions of a 1$\mathrm{D}$ chemotaxis model with volume-filling effect. Their results provided insights on how the biological parameters affect pattern formation.

The chemotaxis models with logistic growth but without a volume-filling effect were studied (see [17-22]). The global attractor and traveling wave solutions of a volume-filling chemotaxis model with logistic growth were obtained in [3] and [23], respectively. Ma et al. [24] studied the existence of stationary solutions of a volume-filling chemotaxis model with logistic cell growth. Moreover, based on an explicit formula for the stationary solutions, which is derived by asymptotic bifurcation analysis, the stability criteria were established and a selection mechanism of the principal wave modes for the stable stationary solution by estimating the leading term of the principal eigenvalue was found. Quite recently, Ma et al. in [25] studied the nonexistence of nonconstant steady state (i.e., stationary pattern) for a chemotaxis model with the volume-filling effect and logistic cell growth and established the critical value of the chemotactic coefficient between the existence and the nonexistence of stationary pattern.

Guo and Hwang [26] investigated nonlinear dynamics near an unstable constant equilibrium in the classical KellerSegel model. Their results can be interpreted as a rigorous mathematical characterization for the early-stage pattern formation in the Keller-Segel model. Very recently, Fu and Liu in [22] and [27] studied instability in the Keller-Segel model with a logistic source and cubic source term, respectively. Their results indicated that chemotaxis-driven nonlinear instability occurs in these models.

In this paper, we mainly consider the nonlinear instability for the following chemotaxis model:

$$
\begin{gathered}
U_{t}=\nabla\left(d_{1} \nabla U-\chi U(1-U) \nabla V\right)+\mu U\left(1-\frac{U}{U_{c}}\right), \\
x \in \mathbb{T}^{d}, \quad t>0, \\
V_{t}=d_{2} \nabla^{2} V+\alpha U-\beta V, \quad x \in \mathbb{T}^{d}, t>0
\end{gathered}
$$

which is subject to the Neumann boundary conditions

$$
\frac{\partial U}{\partial x_{i}}=\frac{\partial V}{\partial x_{i}}=0, \quad \text { at } x_{i}=0, \pi, 1<i<d
$$

and the nonnegative initial data

$$
\begin{array}{r}
U(x, 0)=U_{0}(x) \geq 0, \quad V(x, 0)=V_{0}(x) \geq 0, \\
x \in \mathbb{T}^{d},
\end{array}
$$

where $\mathbb{T}^{d}=(0, \pi)^{d}(d=1,2,3)$ is a $d$-dimensional box. The term $\mu U\left(1-U / U_{c}\right)$ describes the logistic growth of cells with growth rate $\mu>0$ and carrying capacity $U_{c}$ fulfilling $0<U_{c} \leq 1$. Our main result (see Theorem 6) indicates that the nonlinear dynamics near an unstable constant equilibrium points in the classical Keller-Segel model, the KellerSegel model with a logistic source and cubic source term, respectively, and a volume-filling chemotaxis model with logistic source term are almost similar.

The organization of this paper is as follows: in Section 2, we show that the unique positive equilibrium point of (2) without chemotaxis is globally asymptotically stable and cross diffusion cannot induce the instability of the positive equilibrium. In Section 3, we consider the growing modes of (2). In Section 4, we present and prove the Bootstrap lemma, which was first introduced in [28]. In Section 5, for any given general perturbation of magnitude $\delta$, we prove that its nonlinear evolution is dominated by the corresponding linear dynamics along a fixed finite number of fastest growing modes, over a time period of the order $\ln (1 / \delta)$. Each initial perturbation certainly can behave drastically different from another, which gives rise to the richness of patterns. 


\section{Stability of Positive Equilibrium Point of (2) without Chemotaxis}

We first discuss the following corresponding kinetic equations of (2):

$$
\begin{aligned}
& U_{t}=\mu U\left(1-\frac{U}{U_{c}}\right), \quad t>0, \\
& V_{t}=\alpha U-\beta V, \quad t>0 .
\end{aligned}
$$

We use $[\cdot, \cdot]$ to denote a column vector. Evidently, (5) has the unique positive equilibrium point $\overline{\mathbf{W}}=[\bar{U}, \bar{V}]=\left[U_{c}\right.$, $\left.(\alpha / \beta) U_{c}\right]$. For simplicity, we denote $\mathbf{F}(\mathbf{W})=\left[\mu U\left(1-U / U_{c}\right)\right.$, $\alpha U-\beta V]$, and a direct calculation yields

$$
\mathbf{F}_{\mathbf{W}}(\overline{\mathbf{W}})=\left(\begin{array}{cc}
-\mu & 0 \\
\alpha & -\beta
\end{array}\right)
$$

The characteristic polynomial of $\mathbf{F}_{\mathbf{W}}(\overline{\mathbf{W}})$ is $\varphi(\lambda)=(\lambda+$ $\mu)(\lambda+\beta) ;-\mu$ and $-\beta$ are the two roots of $\varphi(\lambda)=0$. Hence $\left[U_{c},(\alpha / \beta) U_{c}\right]$ is locally stable. Define $E(t)=p\left(U-U_{c}-\right.$ $\left.U_{c} \ln \left(U / U_{c}\right)\right)+\left(V-(\alpha / \beta) U_{c}\right)^{2}$, where $p=2 \alpha^{2} U_{c} / \beta \mu$. By the Lyapunov-LaSalle invariance principle $[29],\left[U_{c},(\alpha / \beta) U_{c}\right]$ is globally asymptotically stable.

We now consider system (2) without chemotaxis in the following form:

$$
\begin{gathered}
U_{t}=d_{1} \Delta U+\mu U\left(1-\frac{U}{U_{c}}\right), \quad x \in \mathbb{T}^{d}(d=1,2,3), \\
t>0, \\
V_{t}=d_{2} \Delta V+\alpha U-\beta V, \quad x \in \mathbb{T}^{d}(d=1,2,3), \\
t>0, \\
\frac{\partial U}{\partial x_{i}}=\frac{\partial V}{\partial x_{i}}=0, \quad \text { at } x_{i}=0, \pi, \text { for } 1 \leq i \leq d .
\end{gathered}
$$

Let $0=\mu_{1}<\mu_{2}<\mu_{3}<\cdots$ be the eigenvalues of the operator $-\Delta$ on $\mathbb{T}^{d}(d=1,2,3)$ with the homogeneous Neumann boundary condition and $E\left(\mu_{i}\right)$ the eigenspace corresponding to $\mu_{i}$ in $L^{2}\left(\mathbb{T}^{d}\right)$. Let $X=\left[L^{2}\left(\mathbb{T}^{d}\right)\right]^{2},\left\{\phi_{i j}: j=1, \ldots, \operatorname{dim} E\left(\mu_{i}\right)\right\}$ be an orthonormal basis of $E\left(\mu_{i}\right)$, and $X_{i j}=\left\{\mathbf{c} \cdot \phi_{i j} \mid \mathbf{c} \in \mathbb{R}^{2}\right\}$. Then $X=\oplus_{i=1}^{\infty} X_{i}, X_{i}=\oplus_{j=1}^{\operatorname{dim} E\left(\mu_{i}\right)} X_{i j}$.

Let $\mathfrak{D}=\operatorname{diag}\left(d_{1}, d_{2}\right)$ and $\mathfrak{Q}=\mathfrak{D} \Delta+\mathbf{F}_{\mathbf{W}}(\overline{\mathbf{W}})$. The linearization of (7) at $[\bar{U}, \bar{V}]$ can be expressed by $\mathbf{W}_{t}=\mathfrak{Q}(\mathbf{W}-\overline{\mathbf{W}})$. For each $i \geq 1, X_{i}$ is invariant under the operator $\mathbb{R}$, and $\lambda$ is an eigenvalue of $\mathfrak{Q}$ on $X_{i}$ if and only if it is an eigenvalue of the matrix

$$
-\mu_{i} \mathfrak{D}+\mathbf{F}_{\mathbf{W}}(\overline{\mathbf{W}})=\left(\begin{array}{cc}
-\mu_{i} d_{1}-\mu & 0 \\
\alpha & -\mu_{i} d_{2}-\beta
\end{array}\right) .
$$

Thus, $-\mu_{i} \mathfrak{D}+\mathbf{F}_{\mathbf{W}}(\overline{\mathbf{W}})$ has two negative eigenvalues $-\mu_{i} d_{1}-$ $\mu$ and $-\mu_{i} d_{2}-\beta$. It follows from Theorem 5.1.1 in [30] that $\left[U_{c},(\alpha / \beta) U_{c}\right]$ is locally asymptotically stable.
Let $\mathbf{W}=[U, V]$ be the unique nonnegative global solution. The maximum principle gives

$$
\begin{aligned}
& 0 \leq U(x, t) \leq \max \left\{U_{c}, \sup _{\mathbb{J}^{d}} U_{0}(x)\right\}, \\
& 0 \leq V(x, t) \leq \max \left\{\frac{\alpha}{\beta} U_{c}, \frac{\alpha}{\beta} \sup _{\mathbb{T}^{d}} U_{0}(x), \sup _{\mathbb{T}^{d}} V_{0}(x)\right\} .
\end{aligned}
$$

Moreover, by the strong maximum principle [31], we know that if $U_{0}, V_{0} \geq(\not \equiv) 0$, then $U(x, t)>0, V(x, t)>0$ on $\overline{\mathbb{T}}^{d}$ for all $t>0$.

We define the Lyapunov function

$$
E(t)=\int_{\mathbb{J}^{d}}\left[p\left(U-U_{c}-U_{c} \ln \frac{U}{U_{c}}\right)+\left(V-\frac{\alpha}{\beta} U_{c}\right)^{2}\right] d x
$$

where $p=2 \alpha^{2} U_{c} / \beta \mu$. Then $E(t) \geq 0$ for all $t \geq 0$. Applying (7) and integration by parts, we have

$$
\begin{aligned}
E^{\prime}(t)= & -\int_{\mathbb{T}^{d}}\left\{\frac{p d_{1} U_{c}}{U^{2}}|\nabla U|^{2}+2 d_{2}|\nabla V|^{2}\right\} d x \\
& -\int_{\mathbb{T}^{d}}\left\{\frac{p \mu}{U_{c}}\left(U-U_{c}\right)^{2}-2 \alpha\left(U-U_{c}\right)\left(V-\frac{\alpha}{\beta} U_{c}\right)\right. \\
& \left.+2 \beta\left(V-\frac{\alpha}{\beta} U_{c}\right)^{2}\right\} d x . \\
\leq & -\frac{\alpha^{2}}{\beta} \int_{\mathbb{T}^{d}}\left(U-U_{c}\right)^{2} d x \\
& -\beta \int_{\mathbb{T}^{d}}\left(V-\frac{\alpha}{\beta} U_{c}\right)^{2} d x .
\end{aligned}
$$

By (9), (11), the basic estimates for parabolic equations [31], and Lemma 2.1 in [22] (which is given in [32] in Chinese), we can conclude that

$$
\begin{aligned}
& \lim _{t \rightarrow \infty}\left\|U(\cdot, t)-U_{c}\right\|_{L^{2}\left(\mathbb{T}^{d}\right)}=0, \\
& \lim _{t \rightarrow \infty}\left\|V(\cdot, t)-\frac{\alpha}{\beta} U_{c}\right\|_{L^{2}\left(\mathbb{T}^{d}\right)}=0 .
\end{aligned}
$$

The global asymptotic stability of $\left[U_{c},(\alpha / \beta) U_{c}\right]$ follows from

(12) together with the local stability of $\left[U_{c},(\alpha / \beta) U_{c}\right]$.

Next, we consider that the cross diffusion model

$$
\begin{array}{r}
U_{t}=d_{1} \Delta\left(U+d_{3} U V\right)+\mu U\left(1-\frac{U}{U_{c}}\right), \\
x \in \mathbb{T}^{d}, \quad t>0, \\
V_{t}=d_{2} \Delta V+\alpha U-\beta V, \quad x \in \mathbb{T}^{d}, t>0,
\end{array}
$$




$$
\begin{array}{r}
\frac{\partial U}{\partial x_{i}}=\frac{\partial V}{\partial x_{i}}=0, \quad \text { at } x_{i}=0, \pi, \text { for } 1 \leq i \leq d, \\
U(x, 0)=U_{0}(x) \geq 0, \quad V(x, 0)=V_{0}(x) \geq 0, \\
x \in \mathbb{T}^{d} .
\end{array}
$$

Let $\Phi(\mathbf{W})=\left[d_{1}\left(U+d_{3} U V\right), d_{2} V\right]$. Then the linearizing system (13) at $\overline{\mathbf{W}}$ can be written as

$$
\mathbf{W}_{t}=\left(\Phi_{\mathbf{W}} \Delta+\mathbf{F}_{\mathbf{W}}(\overline{\mathbf{W}})\right) \mathbf{W}
$$

where

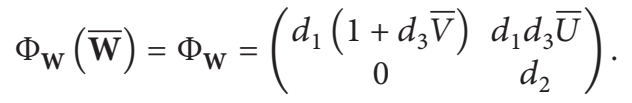

Then, for each $i \in\{1,2, \ldots\}, X_{i}$ is invariant under the operator $\Phi_{\mathbf{W}} \Delta+\mathbf{F}_{\mathbf{W}}(\overline{\mathbf{W}})$, and $\xi$ is an eigenvalue of $\Phi_{\mathbf{W}} \Delta+\mathbf{F}_{\mathbf{W}}(\overline{\mathbf{W}})$ on $X_{i}$ if and only if $\xi$ is an eigenvalue of the matrix

$$
\mathscr{A}_{i}=\left(\begin{array}{cc}
-d_{1}\left(1+d_{3} \bar{V}\right) \mu_{i}-\mu & -d_{1} d_{3} \bar{U} \mu_{i} \\
\alpha & -d_{2} \mu_{i}-\beta
\end{array}\right) .
$$

Notice that

$$
\begin{aligned}
\operatorname{det} \mathscr{A}_{i}= & d_{1} d_{2}\left(1+d_{3} \bar{V}\right) \mu_{i}^{2} \\
& +\left(d_{2} \mu+\beta d_{1}\left(1+d_{3} \bar{V}\right)+d_{1} d_{3} \alpha \bar{U}\right) \mu_{i} \\
& +\mu \beta>0 \\
\operatorname{Tr} \mathscr{A}_{i}= & -\left(\left(d_{1}+d_{2}+d_{1} d_{3} \bar{V}\right) \mu_{i}+\mu+\beta\right)<0
\end{aligned}
$$

Thus, the two eigenvalues $\xi_{i}^{+}$and $\xi_{i}^{-}$of $\mathscr{A}_{i}$ have negative real parts.

From this, we see that adding the cross diffusion to the system (7), the positive constant solution is also locally stable, which means that Turing instability does not occur. The information above indicates that the aggregation of individuals does not occur in the absence of chemotactic effect. It is the purpose of the present paper to clarify the effect of the chemotaxis and nonlinear patterns created by chemotaxis for a volume-filling chemotaxis model with logistic growth.

\section{Growing Modes in the System (2)}

The nonlinear evolution of a perturbation $u(\mathbf{x}, t)=U(\mathbf{x}, t)-$ $\bar{U}, v(\mathbf{x}, t)=V(\mathbf{x}, t)-\bar{V}$ around $[\bar{U}, \bar{V}]$ satisfies

$$
\begin{aligned}
u_{t}= & d_{1} \nabla^{2} u-\chi U_{c}\left(1-U_{c}\right) \nabla^{2} v-\mu u \\
& -\chi \nabla\left[u\left(1-u-2 U_{c}\right) \nabla v\right]-\frac{\mu u^{2}}{U_{c}}, \\
v_{t}= & d_{2} \nabla^{2} v+\alpha u-\beta v .
\end{aligned}
$$

The corresponding linearized system is as follows:

$$
\begin{aligned}
& u_{t}=d_{1} \nabla^{2} u-\chi U_{c}\left(1-U_{c}\right) \nabla^{2} v-\mu u, \\
& v_{t}=d_{2} \nabla^{2} v+\alpha u-\beta v .
\end{aligned}
$$

Let $\mathbf{w}(\mathbf{x}, t) \equiv[u(\mathbf{x}, t), v(\mathbf{x}, t)], \mathbf{q}=\left(q_{1}, \ldots, q_{d}\right) \in \Omega=$ $(\mathbb{N} \cup\{0\})^{d}$, and $e_{\mathbf{q}}(\mathbf{x})=\prod_{i=1}^{d} \cos \left(q_{i} x_{i}\right)$. Then $\left\{e_{\mathbf{q}}(\mathbf{x})\right\}_{\mathbf{q} \in \Omega}$ forms a basis of the space of functions in $\mathbb{T}^{d}$ that satisfies Neumann boundary conditions. We try to find a normal mode to the linear system (19) of the following form:

$$
\mathbf{w}(\mathbf{x}, t)=\mathbf{r}_{\mathbf{q}} e^{\lambda_{\mathbf{q}} t} e_{\mathbf{q}}(\mathbf{x}),
$$

where $\mathbf{r}_{\mathbf{q}}$ is a vector depending on $\mathbf{q}$. Substituting (20) into (19) yields

$$
\lambda_{\mathbf{q}} \mathbf{r}_{\mathbf{q}}=\left(\begin{array}{cc}
-d_{1} q^{2}-\mu & \chi U_{c}\left(1-U_{c}\right) q^{2} \\
\alpha & -d_{2} q^{2}-\beta
\end{array}\right) \mathbf{r}_{\mathbf{q}},
$$

where $q^{2}=\sum_{i=1}^{d} q_{i}^{2}$. We can obtain the following dispersion formula for $\lambda_{\mathbf{q}}$ :

$$
\begin{aligned}
\lambda_{\mathbf{q}}^{2} & +\left(\left(d_{1}+d_{2}\right) q^{2}+\beta+\mu\right) \lambda_{\mathbf{q}} \\
& +q^{2}\left[d_{1} d_{2} q^{2}+\left(d_{1} \beta+d_{2} \mu-\alpha \chi U_{c}\left(1-U_{c}\right)\right)\right] \\
& +\mu \beta=0 .
\end{aligned}
$$

Thus, we deduce the following linear instability criterion by requiring that there exist a $\mathbf{q}$ such that

$$
q^{2}\left[d_{1} d_{2} q^{2}+\left(d_{1} \beta+d_{2} \mu-\alpha \chi U_{c}\left(1-U_{c}\right)\right)\right]+\mu \beta<0
$$

to ensure that (22) has at least one positive root $\lambda_{\mathbf{q}}$. This means that

$$
\left(q^{2}\left(d_{1}-d_{2}\right)-\beta+\mu\right)^{2}+4 \alpha \chi U_{c}\left(1-U_{c}\right) q^{2}>0 .
$$

There exist two distinct real roots:

$$
\begin{aligned}
\lambda_{\mathbf{q}}^{ \pm} & \\
= & \left(-\left(q^{2}\left(d_{1}+d_{2}\right)+\beta+\mu\right)\right. \\
& \left. \pm \sqrt{\left(q^{2}\left(d_{1}-d_{2}\right)-\beta+\mu\right)^{2}+4 \alpha \chi U_{c}\left(1-U_{c}\right) q^{2}}\right) \\
& \times(2)^{-1}
\end{aligned}
$$

for all $\mathbf{q} \neq \mathbf{0}$ to the quadratic equation (22), which are denoted by $\lambda_{\mathbf{q}}^{-}<\lambda_{\mathbf{q}}^{+}$. We denote the corresponding (linearly independent) eigenvectors by $\mathbf{r}_{-}(\mathbf{q})$ and $\mathbf{r}_{+}(\mathbf{q})$, such that

$$
\mathbf{r}_{ \pm}(\mathbf{q})=\left[\frac{\lambda_{\mathbf{q}}^{ \pm}+d_{2} q^{2}+\beta}{\alpha}, 1\right] .
$$

Clearly, for $q$ large,

$$
q^{2}\left[d_{1} d_{2} q^{2}+\left(d_{1} \beta+d_{2} \mu-\alpha \chi U_{c}\left(1-U_{c}\right)\right)\right]+\mu \beta>0 .
$$

Thus, there are only finitely many $\mathbf{q}$ such that $\lambda_{\mathbf{q}}^{+}>0$. We denote the largest eigenvalue by $\lambda_{\max }>0$ and define 
$\Omega_{\max } \equiv\left\{\mathbf{q} \in \Omega \mid \lambda_{\mathbf{q}}^{+}=\lambda_{\max }\right\}$. From (25) we can regard $\lambda_{\mathbf{q}}^{+}$as a function of $q^{2}$. Therefore, there is one $q^{2}$ (possibly two) having $\lambda^{+}\left(q^{2}\right)=\lambda_{\text {max }}$. We also denote $\rho>0$ to be the gap between the $\lambda_{\text {max }}$ and the rest; that is, $\rho=\min _{\mathbf{q} \in \Omega \backslash \Omega_{\max }}\left|\lambda_{\max }-\lambda_{\mathbf{q}}\right|$. as

Given any initial perturbation $\mathbf{w}(\mathbf{x}, 0)$, we can expand it

$$
\mathbf{w}(\mathbf{x}, 0)=\sum_{\mathbf{q} \in \Omega} \mathbf{w}_{\mathbf{q}} e_{\mathbf{q}}(\mathbf{x})=\sum_{\mathbf{q} \in \Omega}\left\{w_{\mathbf{q}}^{-} \mathbf{r}_{-}(\mathbf{q})+w_{\mathbf{q}}^{+} \mathbf{r}_{+}(\mathbf{q})\right\} e_{\mathbf{q}}(\mathbf{x}),
$$

$$
\mathbf{w}_{\mathbf{q}}=w_{\mathbf{q}}^{-} \mathbf{r}_{-}(\mathbf{q})+w_{\mathbf{q}}^{+} \mathbf{r}_{+}(\mathbf{q})
$$

In the sequel, denote by $\langle\cdot, \cdot\rangle$ and $(\cdot, \cdot)$ the inner product of $\left[L^{2}\left(\mathbb{T}^{d}\right)\right]^{2}$ and the scalar product of $\mathbb{R}^{2}$, respectively. For any $\mathbf{g}(\cdot, t) \in\left[L^{2}\left(\mathbb{T}^{d}\right)\right]^{2}$, we denote $\|\mathbf{g}(\cdot, t)\| \equiv\|\mathbf{g}(\cdot, t)\|_{L^{2}}$. Throughout this paper, we always denote universal constants by $C_{i}(i=1,2, \ldots)$.

Clearly,

$$
\|\mathbf{w}(\mathbf{x}, 0)\|^{2}=\left(\frac{\pi}{2}\right)^{d} \sum_{\mathbf{q} \in \Omega}\left|\mathbf{w}_{\mathbf{q}}\right|^{2}
$$

The unique solution $\mathbf{w}(\mathbf{x}, t)=[u(\mathbf{x}, t), v(\mathbf{x}, t)]$ of $(19)$ is given by

$$
\begin{aligned}
\mathbf{w}(\mathbf{x}, t) & =\sum_{\mathbf{q} \in \Omega}\left\{w_{\mathbf{q}}^{-} \mathbf{r}_{-}(\mathbf{q}) e^{\lambda_{\mathbf{q}}^{-} t}+w_{\mathbf{q}}^{+} \mathbf{r}_{+}(\mathbf{q}) e^{\lambda_{\mathbf{q}}^{+} t}\right\} e_{\mathbf{q}}(\mathbf{x}) \\
& \equiv e^{\mathfrak{Q} t} \mathbf{W}(\mathbf{x}, 0) .
\end{aligned}
$$

Our main result in this section is the following lemma.

Lemma 1. Suppose that the instability criterion (23) holds. Let $\mathbf{w}(\mathbf{x}, t) \equiv e^{\mathfrak{\Omega} t} \mathbf{w}(\mathbf{x}, 0)$ be a solution to the linearized system (19) with initial condition $\mathbf{w}(\mathbf{x}, 0)$. Then there exists a constant $\widehat{C}_{1} \geq$ 1 depending on $d_{1}, d_{2}, U_{c}, \chi, \mu, \alpha$, and $\beta$, such that

$$
\|\mathbf{w}(\cdot, t)\| \leq \widehat{C}_{1} e^{\lambda_{\max } t}\|\mathbf{w}(\cdot, 0)\|, \quad \forall t \geq 0 .
$$

Proof. We divide the proof into the following two cases.

(1) $t \geq 1$. It follows from (25) that

$$
\lim _{\mathbf{q} \rightarrow \infty} \frac{\lambda_{\mathbf{q}}^{ \pm}}{q^{2}}=-d_{1},-d_{2}
$$

respectively. Thus, $\lambda_{\mathbf{q}}^{ \pm} \leq-\min \left\{d_{1}, d_{2}\right\} q^{2}$ and there exists a positive constant $C_{1}$ for all $\mathbf{q}>0$, such that

$$
\left|\frac{\lambda_{\mathbf{q}}^{ \pm}}{q^{2}}\right| \leq C_{1}
$$

By the quadratic formula of (22), one can obtain

$$
\left|\lambda_{\mathbf{q}}^{+}-\lambda_{\mathbf{q}}^{-}\right| \geq 2 q \sqrt{\alpha \chi U_{c}\left(1-U_{c}\right)} .
$$

It follows from (29) that

$$
\left|w_{\mathbf{q}}^{ \pm}\right| \leq \frac{\left|\mathbf{r}_{ \pm}(\mathbf{q})\right| \times\left|\mathbf{w}_{\mathbf{q}}\right|}{\left|\operatorname{det}\left[\mathbf{r}_{-}(\mathbf{q}), \mathbf{r}_{+}(\mathbf{q})\right]\right|}
$$

From (26) and (34), we can conclude that there exists a positive constant $C_{2}$ such that

$$
\left|\mathbf{r}_{ \pm}(\mathbf{q})\right|=\sqrt{\left[\left(\frac{\lambda_{\mathbf{q}}^{ \pm}}{\alpha q^{2}}+\frac{d_{2}}{\alpha}\right) q^{2}+\frac{\beta}{\alpha}\right]^{2}+1} \leq C_{2} q^{2}
$$

for all $q>0$, where $C_{2}=2 \max \left\{C_{1}+d_{2} / \alpha, 1+\beta / \alpha\right\}$. By (34), one can deduce

$$
\frac{1}{\left|\operatorname{det}\left[\mathbf{r}_{-}(\mathbf{q}), \mathbf{r}_{+}(\mathbf{q})\right]\right|} \leq \frac{1}{2 q} \sqrt{\frac{\alpha}{\chi U_{c}\left(1-U_{c}\right)}} .
$$

Combining (37) and (38), we find that

$$
\left|w_{\mathbf{q}}^{ \pm}\right| \leq C_{3} q\left|\mathbf{w}_{\mathbf{q}}\right|
$$

where $C_{3}=\left(C_{2} / 2\right) \sqrt{\alpha / \chi U_{c}\left(1-U_{c}\right)}$. For $t \geq 1$ and $q$ large, it is not difficult to verify by (33), (37), and (39) that

$$
\begin{aligned}
\left|w_{\mathbf{q}}^{ \pm} \mathbf{r}_{ \pm}(\mathbf{q}) e^{\lambda_{\mathbf{q}}^{ \pm} t}\right| & \leq C_{2} C_{3} q^{3}\left|\mathbf{w}_{\mathbf{q}}\right| e^{\lambda_{\mathbf{q}}^{ \pm} t} \\
& \leq C_{4}\left|\mathbf{w}_{\mathbf{q}}\right| \frac{q^{3}}{\exp \left(\min \left\{d_{1}, d_{2}\right\} q^{2}\right)} \leq C_{5}\left|\mathbf{w}_{\mathbf{q}}\right|
\end{aligned}
$$

In view of (30) and (40), we observe that

$$
\|\mathbf{w}(\mathbf{x}, t)\| \leq 2 C_{5} e^{\lambda_{\max } t}\|\mathbf{w}(\mathbf{x}, 0)\|, \quad \text { for } t \geq 1 .
$$

(2) $t \leq 1$. Multiplying the first equation of (19) by $u$ and the second by $K v$, adding them together, and integrating the result in $\mathbb{T}^{d}$, we have

$$
\begin{aligned}
& \frac{1}{2} \frac{d}{d t} \int_{\mathbb{T}^{d}}\left\{|u|^{2}+K|v|^{2}\right\} \mathbf{d} \mathbf{x} \\
& +\int_{\mathbb{J}^{d}}\left\{d_{1}|\nabla u|^{2}+K d_{2}|\nabla v|^{2}\right. \\
& \left.\quad-\chi U_{c}\left(1-U_{c}\right) \nabla u \nabla v\right\} \mathbf{d} \mathbf{x} \\
& =-\mu \int_{\mathbb{T}^{d}} u^{2} \mathbf{d x}-K \beta \int_{\mathbb{T}^{d}} v^{2} \mathbf{d} \mathbf{x} \\
& +\alpha K \int_{\mathbb{T}^{d}} u v \mathbf{d x} .
\end{aligned}
$$

Let

$$
K=\frac{\left[\chi U_{c}\left(1-U_{c}\right)\right]^{2}}{d_{1} d_{2}} .
$$


Then the integrand of the second integral can be estimated as follows:

$$
\begin{gathered}
d_{1}|\nabla u|^{2}+K d_{2}|\nabla v|^{2}-\chi U_{c}\left(1-U_{c}\right) \nabla u \nabla v \\
\geq \frac{d_{1}}{2}|\nabla u|^{2}+\frac{\left[\chi U_{c}\left(1-U_{c}\right)\right]^{2}}{2 d_{1}}|\nabla v|^{2} \geq 0 .
\end{gathered}
$$

Using Young inequality, we deduce that

$$
\frac{1}{2} \frac{d}{d t} \int_{\mathbb{T}^{d}}\left\{|u|^{2}+K\left|v^{2}\right|\right\} \mathbf{d} \mathbf{x} \leq \frac{\alpha \sqrt{K}}{2} \int_{\mathbb{T}^{d}}\left\{|u|^{2}+K|v|^{2}\right\} \mathbf{d x} .
$$

It follows from Gronwall inequality that

$$
\begin{aligned}
& \int_{\mathbb{T}^{d}}\left\{|u(\mathbf{x}, t)|^{2}+K|v(\mathbf{x}, t)|^{2}\right\} \mathbf{d} \mathbf{x} \\
& \leq e^{\alpha \sqrt{K} t} \int_{\mathbb{T}^{d}}\left\{|u(\mathbf{x}, t)|^{2}+K|v(\mathbf{x}, t)|^{2}\right\} \mathbf{d x} .
\end{aligned}
$$

If $K \geq 1$ and $t \leq 1$, then it is clear from (46) that

$$
\|\mathbf{w}(\mathbf{x}, t)\| \leq \sqrt{K e^{\alpha \sqrt{K}}} e^{\lambda_{\max } t}\|\mathbf{w}(\mathbf{x}, 0)\|
$$

Similarly, if $0<K<1, t \leq 1$, then from (46), one has

$$
\|\mathbf{w}(\mathbf{x}, t)\| \leq \sqrt{e^{\alpha} / K} e^{\lambda_{\max } t}\|\mathbf{w}(\mathbf{x}, 0)\|
$$

Let $\widehat{C}_{1}=\max \left\{2 C_{5}, \sqrt{e^{\alpha} / K}\right\} \geq 1$ if $0<K<1$, and let $\widehat{C}_{1}=$ $\max \left\{2 C_{5}, \sqrt{K e^{\alpha \sqrt{K}}}\right\} \geq 1$ if $K \geq 1$. Then

$$
\|\mathbf{w}(\mathbf{x}, t)\| \leq \widehat{C}_{1} e^{\lambda_{\max } t}\|\mathbf{w}(\mathbf{x}, 0)\|
$$

This completes the proof of Lemma 1.

\section{Bootstrap Lemma}

By a standard PDE theory [31], we can establish the existence of local solutions for (18).

Lemma 2 (Local existence). For $s \geq 1(d=1)$ and $s \geq 2(d=$ $2,3)$, there exists a $T_{0}>0$ such that (18) with $u(\cdot, 0), v(\cdot, 0) \in$ $H^{s}$ has a unique solution $\mathbf{w}(\cdot, t)$ on $\left(0, T_{0}\right)$ which satisfies

$$
\|\mathbf{w}(t)\|_{H^{s}} \leq C\|\mathbf{w}(0)\|_{H^{s}}, \quad 0<t<T_{0},
$$

where $C$ is a positive constant depending on $d_{1}, d_{2}, U_{c}, \alpha, \beta$, and $\chi$.

It is not difficult to verify the following result.

Lemma 3. Let $\mathbf{w}(\mathbf{x}, t)$ be a solution of (18). Then the even extension of $\mathbf{w}(\mathbf{x}, t)$ on $2 \mathbb{T}^{d}=(-\pi, \pi)^{d}(d=1,2,3)$ is also the solution of (18) which satisfies homogeneous Neumann boundary conditions and periodical boundary conditions on $2 \mathbb{T}^{d}=(-\pi, \pi)^{d}(d=1,2,3)$.
Lemma 4. Let $[u(\mathbf{x}, t), v(\mathbf{x}, t)]$ be a solution of (18). Then

$$
\begin{aligned}
& \frac{1}{2} \frac{d}{d t} \sum_{|\alpha|=2} \int_{\mathbb{T}^{d}}\left\{\left|D^{\alpha} u\right|^{2}+\frac{\left[\chi U_{c}\left(1-U_{c}\right)\right]^{2}}{d_{1} d_{2}}\left|D^{\alpha} v\right|^{2}\right\} \mathbf{d x} \\
& \quad+\sum_{|\alpha|=2} \int_{\mathbb{T}^{d}}\left\{\frac{d_{1}}{4}\left|\nabla D^{\alpha} u\right|^{2}+\frac{\left[\chi U_{c}\left(1-U_{c}\right)\right]^{2}}{2 d_{1}}\left|\nabla D^{\alpha} v\right|^{2}\right\} \mathbf{d} \mathbf{x} \\
& \quad+\mu \sum_{|\alpha|=2} \int_{\mathbb{T}^{d}}\left|D^{\alpha} u\right|^{2} \mathbf{d x}+\frac{\beta\left[\chi U_{c}\left(1-U_{c}\right)\right]^{2}}{2 d_{1} d_{2}} \\
& \quad \times \sum_{|\alpha|=2} \int_{\mathbb{T}^{d}}\left|D^{\alpha} v\right|^{2} \mathbf{d x} \\
& \leq \widehat{C}_{2}\left(\|\mathbf{w}\|_{H^{2}}+\|\mathbf{w}\|_{H^{2}}^{2}\right)\left\|\nabla^{3} \mathbf{w}\right\|^{2}+\widehat{C}_{3}\|u\|^{2},
\end{aligned}
$$

where $\widehat{C}_{3}=C_{0}^{3} \alpha^{3}\left[\chi U_{c}\left(1-U_{c}\right)\right]^{6} / 2 \beta^{3} d_{1}^{5} d_{2}^{3}$.

Proof. It is known by Lemma 3 that

$$
\begin{gathered}
\tilde{u}_{t}=d_{1} \nabla^{2} \tilde{u}-\chi U_{c}\left(1-U_{c}\right) \nabla^{2} \widetilde{v}-\mu \tilde{u} \\
-\chi \nabla\left[\widetilde{u}\left(1-\tilde{u}-2 U_{c}\right) \nabla \widetilde{v}\right]-\frac{\mu \tilde{u}^{2}}{U_{c}}, \\
x \in 2 \mathbb{T}^{d}, \quad t>0, \\
\widetilde{v}_{t}=d_{2} \nabla^{2} \widetilde{v}+\alpha \widetilde{u}-\beta \widetilde{v}, \quad x \in 2 \mathbb{T}^{d}, t>0, \\
\frac{\partial \widetilde{u}}{\partial x_{i}}=\frac{\partial \widetilde{v}}{\partial x_{i}}=0, \quad \text { at } x_{i}=-\pi, 0, \pi, \text { for } 1 \leq i \leq d,
\end{gathered}
$$

where $[\widetilde{u}(\mathbf{x}, t), \widetilde{v}(\mathbf{x}, t)]$ is the even extension of $[u(\mathbf{x}, t), v(\mathbf{x}, t)]$ on $2 \mathbb{T}^{d}$. Taking the second-order derivative of (52) for $x_{i}, x_{j}$ and making inner product with $\partial_{x_{i} x_{j}} \widetilde{u}$ and $K \partial_{x_{i} x_{j}} \widetilde{v}$, respectively, and adding them together, we deduce that

$$
\begin{aligned}
& \frac{1}{2} \frac{d}{d t} \int_{2 \mathbb{J}^{d}}\left\{\left|\partial_{x_{i} x_{j}} \widetilde{u}\right|^{2}+K\left|\partial_{x_{i} x_{j}} \widetilde{v}\right|^{2}\right\} \mathbf{d x} \\
& +\int_{2 \mathbb{J}^{d}}\left\{d_{1}\left|\nabla \partial_{x_{i} x_{j}} \tilde{u}\right|^{2}+K d_{2}\left|\nabla \partial_{x_{i} x_{j}} \widetilde{v}\right|^{2}\right. \\
& \left.-\chi U_{c}\left(1-U_{c}\right) \nabla \partial_{x_{i} x_{j}} \tilde{u} \cdot \nabla \partial_{x_{i} x_{j}} \widetilde{v}\right\} \mathbf{d} \mathbf{x} \\
& +\mu \int_{2 \mathbb{T}^{d}}\left|\partial_{x_{i} x_{j}} \widetilde{u}\right|^{2} \mathbf{d x}+\beta K \int_{2 \mathbb{T}^{d}}\left|\partial_{x_{i} x_{j}} \widetilde{v}\right|^{2} \mathbf{d x} \\
& =\chi\left(1-2 U_{c}\right) \int_{2 \mathbb{T}^{d}} \nabla \partial_{x_{i} x_{j}} \widetilde{u} \cdot \partial_{x_{i} x_{j}}(\widetilde{u} \cdot \nabla \widetilde{v}) \mathbf{d x} \\
& -\chi \int_{2 \mathbb{\Psi}^{d}} \nabla \partial_{x_{i} x_{j}} \tilde{u} \cdot \partial_{x_{i} x_{j}}\left(\widetilde{u}^{2} \nabla \widetilde{v}\right) \mathbf{d} \mathbf{x} \\
& +K \alpha \int_{2 \mathbb{T}^{d}} \partial_{x_{i} x_{j}} \tilde{u} \cdot \partial_{x_{i} x_{j}} \widetilde{v} \mathbf{d x} \\
& -\frac{2 \mu}{U_{c}} \int_{2 \mathbb{T}^{d}}\left[\tilde{u}\left(\partial_{x_{i} x_{j}} \tilde{u}\right)^{2}+\partial_{x_{i}} \tilde{u} \cdot \partial_{x_{j}} \tilde{u} \cdot \partial_{x_{i} x_{j}} \tilde{u}\right] \mathbf{d x} \\
& \equiv I_{1}+I_{2}+I_{3}+I_{4} \text {. }
\end{aligned}
$$


We can apply Young inequality and (43) to get

$$
\begin{aligned}
& d_{1}\left|\nabla \partial_{x_{i} x_{j}} \tilde{u}\right|^{2}+K d_{2}\left|\nabla \partial_{x_{i} x_{j}} \widetilde{v}\right|^{2} \\
& \quad-\chi U_{c}\left(1-U_{c}\right) \nabla \partial_{x_{i} x_{j}} \tilde{u} \cdot \nabla \partial_{x_{i} x_{j}} \widetilde{v} \\
& \geq \frac{d_{1}}{2}\left|\nabla \partial_{x_{i} x_{j}} \tilde{u}\right|^{2}+\frac{\left[\chi U_{c}\left(1-U_{c}\right)\right]^{2}}{2 d_{1}}\left|\nabla \partial_{x_{i} x_{j}} \widetilde{v}\right|^{2} .
\end{aligned}
$$

Now we estimate each term on the right-hand sides of (54). By using Hölder inequality,

$$
\begin{aligned}
& I_{1} \leq \chi\left|1-2 U_{c}\right| \\
& \times\left\{\|\nabla \widetilde{v}\|_{L^{\infty}}\left\|\nabla \partial_{x_{i} x_{j}} \widetilde{u}\right\|\left\|\partial_{x_{i} x_{j}} \widetilde{u}\right\|\right. \\
& +2 \sum_{i=1}^{d}\|\nabla \widetilde{u}\|_{L^{\infty}}\left\|\partial_{x_{i} x_{j}} \widetilde{v}\right\|\left\|\nabla \partial_{x_{i} x_{j}} \widetilde{u}\right\| \\
& \left.+\|\widetilde{u}\|_{L^{\infty}}\left\|\nabla \partial_{x_{i} x_{j}} \widetilde{u}\right\|\left\|\nabla \partial_{x_{i} x_{j}} \widetilde{v}\right\|\right\} .
\end{aligned}
$$

Notice that

$$
\begin{aligned}
& \|g\|_{L^{\infty}\left(2 \mathbb{T}^{d}\right)} \leq C_{6}\|g\|_{H^{2}\left(2 \mathbb{T}^{d}\right)}, \\
& \|g\|_{L^{4}\left(2 \mathbb{T}^{d}\right)} \leq C_{7}\|g\|_{H^{2}\left(2 \mathbb{T}^{d}\right)}, \\
& \|g\|_{L^{6}\left(2 \mathbb{T}^{d}\right)} \leq C_{8}\|g\|_{H^{2}\left(2 \mathbb{T}^{d}\right)},
\end{aligned}
$$

for $d \leq 3$. Clearly,

$$
\begin{aligned}
& \int_{2 \mathbb{\Psi}^{d}} \nabla \widetilde{u} d \mathbf{x}=\int_{2 \mathbb{\Psi}^{d}} \nabla \widetilde{v} d \mathbf{x}=0, \\
& \int_{2 \mathbb{J}^{d}} \partial_{x_{i} x_{j}} \tilde{u} d \mathbf{x}=\int_{2 \mathbb{J}^{d}} \partial_{x_{i} x_{j}} \widetilde{v} d \mathbf{x}=0 .
\end{aligned}
$$

Using the Poincaré inequality, we have

$$
\begin{aligned}
\|g\| & \leq C_{9}\|g\|_{L^{4}\left(2 \mathbb{T}^{d}\right)} \leq C_{10}\|g\|_{L^{6}\left(2 \mathbb{T}^{d}\right)} \\
& \leq C_{11}\|g\|_{H^{1}} \leq C_{12}\|\nabla g\|, \quad d \leq 3 .
\end{aligned}
$$

It follows from (58) and (59) that

$$
\begin{aligned}
& \left\|\partial_{x_{i}} g\right\| \leq C_{12}\left\|\nabla \partial_{x_{i}} g\right\|, \quad\left\|\partial_{x_{i} x_{j}} g\right\| \leq C_{12}\left\|\nabla \partial_{x_{i} x_{j}} g\right\|, \\
& \|\nabla g\| \leq C_{12}\left(\sum_{|\alpha|=2}\left\|D^{\alpha} g\right\|^{2}\right)^{1 / 2} \leq C_{12}^{2}\left(\sum_{|\alpha|=2}\left\|\nabla D^{\alpha} g\right\|^{2}\right)^{1 / 2} .
\end{aligned}
$$

Furthermore,

$$
\begin{aligned}
& \|\nabla g\|_{H^{2}} \leq C_{13}\left(\sum_{|\alpha|=2}\left\|\nabla D^{\alpha} g\right\|^{2}\right)^{1 / 2}, \\
& C_{13}=\left(C_{12}^{4}+C_{12}^{2}+1\right)^{1 / 2} .
\end{aligned}
$$

Combining (56), (57), and (62), we observe that

$$
\sum_{|\alpha|=2} I_{1} \leq \chi\left|1-2 U_{c}\right| C_{14}\|\widetilde{\mathbf{w}}\|_{H^{2}}\left\|\nabla^{3} \widetilde{\mathbf{w}}\right\|^{2},
$$

where $C_{14}=C_{6}\left[(1+2 d) C_{13}+1\right]$.

By Hölder inequality, it follows from (57) and (62) that

$$
\sum_{|\alpha|=2} I_{2} \leq \chi C_{15}\|\widetilde{\mathbf{w}}\|_{H^{2}}^{2}\left\|\nabla^{3} \widetilde{\mathbf{w}}\right\|^{2}
$$

where $C_{15}=C_{6}\left(2 C_{9}^{-2} C_{12}^{2} C_{13}+6 C_{6} C_{13}+C_{6}\right)$.

Now we estimate $I_{3}$. By interpolation for all $a>0$, it can be proved that

$$
\left\|\partial_{x_{i} x_{j}} \widetilde{u}\right\|^{2} \leq C_{0}\left(a\left\|\nabla \partial_{x_{i} x_{j}} \widetilde{u}\right\|^{2}+\frac{\|\widetilde{u}\|^{2}}{4 a^{2}}\right) .
$$

Then it is easy to see that

$$
\begin{aligned}
\sum_{|\alpha|=2} I_{3} \leq & \frac{K \beta}{2} \sum_{|\alpha|=2} \int_{2 \mathbb{J}^{d}}\left|D^{\alpha} \widetilde{v}\right|^{2} \mathbf{d x} \\
& +\frac{d_{1}}{4} \sum_{|\alpha|=2} \int_{2 \mathbb{J}^{d}}\left|\nabla D^{\alpha} \tilde{u}\right|^{2} \mathbf{d} \mathbf{x}+\widehat{C}_{3}\|\widetilde{u}\|^{2},
\end{aligned}
$$

where $\widehat{C}_{3}=C_{0}^{3} \alpha^{3}\left[\chi U_{c}\left(1-U_{c}\right)\right]^{6} / 2 \beta^{3} d_{1}^{5} d_{2}^{3}$ and $a=d_{1}^{2} d_{2} \beta /$ $2\left[\chi U_{c}\left(1-U_{c}\right)\right]^{2} C_{0} \alpha$.

Finally, from (57) and (61), $I_{4}$ is bounded by

$$
\sum_{|\alpha|=2} I_{4} \leq \frac{4 \mu}{U_{c}} C_{16}\|\widetilde{\mathbf{w}}\|_{H^{2}}\left\|\nabla^{3} \widetilde{\mathbf{w}}\right\|^{2} .
$$

Combining (54), (55), and (63)-(67), one can obtain

$$
\begin{aligned}
& \frac{1}{2} \frac{d}{d t} \sum_{|\alpha|=2} \int_{\mathbb{T}^{d}}\left\{\left|D^{\alpha} u\right|^{2}+K\left|D^{\alpha} v\right|^{2}\right\} \mathbf{d} \mathbf{x} \\
& \quad+\sum_{|\alpha|=2} \int_{\mathbb{T}^{d}}\left\{\frac{d_{1}}{4}\left|\nabla D^{\alpha} u\right|^{2}+\frac{K d_{2}}{2}\left|\nabla D^{\alpha} v\right|^{2}\right\} \mathbf{d x} \\
& \quad+\mu \sum_{|\alpha|=2} \int_{\mathbb{T}^{d}}\left|D^{\alpha} u\right|^{2} \mathbf{d x}+\frac{\beta K}{2} \sum_{|\alpha|=2} \int_{\mathbb{T}^{d}}\left|D^{\alpha} v\right|^{2} \mathbf{d x} \mathbf{x} \\
& \leq \widehat{C}_{2}\left(\|\mathbf{w}\|_{H^{2}}+\|\mathbf{w}\|_{H^{2}}^{2}\right)\left\|\nabla^{3} \mathbf{w}\right\|^{2}+\widehat{C}_{3}\|u\|^{2},
\end{aligned}
$$

where $\widehat{C}_{2}=\left(\chi\left|1-2 U_{c}\right|+\chi+\left(4 \mu / U_{c}\right)\right) \max \left\{C_{14}, C_{15}, C_{16}\right\}$ and the proof is completed.

Lemma 5. Let $\mathbf{w}(\mathbf{x}, t)$ be a solution of (18) such that for $0 \leq$ $t \leq T<T_{0}$,

$$
\begin{aligned}
\|\mathbf{w}(\cdot, t)\|_{H^{2}}+\|\mathbf{w}(\cdot, t)\|_{H^{2}}^{2} & \leq \frac{1}{\widehat{C}_{2}} \min \left\{\frac{d_{1}}{4}, \frac{\left[\chi U_{c}\left(1-U_{c}\right)\right]^{2}}{2 d_{1}}\right\}, \\
\|\mathbf{w}(\cdot, t)\| & \leq 2 \widehat{C}_{1} e^{\lambda_{\max } t}\|\mathbf{w}(\cdot, 0)\| .
\end{aligned}
$$


Then

$$
\begin{array}{r}
\|\mathbf{w}(\cdot, t)\|_{H^{2}}^{2} \leq \widehat{C}_{4}\left\{\|\mathbf{w}(\cdot, 0)\|_{H^{2}}^{2}+e^{2 \lambda_{\max } t}\|\mathbf{w}(\cdot, 0)\|^{2}\right\}, \\
0 \leq t \leq T,
\end{array}
$$

where $\widehat{C}_{4}=\max \left\{\left(1+C_{12}^{2}\right)\left[\chi U_{c}\left(1-U_{c}\right)\right]^{2} / d_{1} d_{2}, 4 \widehat{C}_{1}^{2}[1+\right.$ $\left.\left.\widehat{C}_{3}\left(1+C_{12}^{2}\right) / \lambda_{\max }\right]\right\}$ if $\left[\chi U_{c}\left(1-U_{c}\right)\right]^{2} / d_{1} d_{2} \geq 1$ and $\widehat{C}_{4}=$ $\max \left\{\left(1+C_{12}^{2}\right) d_{1} d_{2} /\left[\chi U_{c}\left(1-U_{c}\right)\right]^{2}, 4 \widehat{C}_{1}^{2}\left[1+\widehat{C}_{3}\left(1+C_{12}^{2}\right) d_{1} d_{2} /\right.\right.$ $\left.\left.\lambda_{\max }\left[\chi U_{c}\left(1-U_{c}\right)\right]^{2}\right]\right\}$ if $\left[\chi U_{c}\left(1-U_{c}\right)\right]^{2} / d_{1} d_{2}<1$.

Proof. It follows from (61) that

$$
\|\nabla \mathbf{w}(\cdot, t)\|^{2} \leq C_{12}^{2} \sum_{|\alpha|=2}\left\|D^{\alpha} \mathbf{w}(\cdot, t)\right\|^{2} .
$$

Thus,

$$
\|\mathbf{w}(\cdot, t)\|_{H^{2}}^{2} \leq\|\mathbf{w}(\cdot, t)\|^{2}+\left(C_{12}^{2}+1\right) \sum_{|\alpha|=2}\left\|D^{\alpha} \mathbf{w}(\cdot, t)\right\|^{2} .
$$

Now we estimate the second-order derivatives of $\mathbf{w}(\cdot, t)$. From Lemma 4 and (69), we can obtain

$$
\begin{aligned}
& \frac{1}{2} \frac{d}{d t} \sum_{|\alpha|=2} \int_{\mathbb{T}^{d}}\left\{\left|D^{\alpha} u\right|^{2}+\frac{\left[\chi U_{c}\left(1-U_{c}\right)\right]^{2}}{d_{1} d_{2}}\left|D^{\alpha} v\right|^{2}\right\} \mathbf{d x} \\
& \quad \leq \widehat{C}_{3}\|u\|^{2} \leq \widehat{C}_{3}\|\mathbf{w}(\cdot, t)\|^{2} .
\end{aligned}
$$

Integrating this from 0 to $t$, we deduce from (70) that

$$
\begin{gathered}
\sum_{|\alpha|=2} \int_{\mathbb{V}^{d}}\left\{\left|D^{\alpha} u(\cdot, t)\right|^{2}+\frac{\left[\chi U_{c}\left(1-U_{c}\right)\right]^{2}}{d_{1} d_{2}}\left|D^{\alpha} v(\cdot, t)\right|^{2}\right\} \mathbf{d x} \\
\leq \sum_{|\alpha|=2} \int_{\mathbb{J}^{d}}\left\{\left|D^{\alpha} u(\cdot, 0)\right|^{2}\right. \\
\left.+\frac{\left[\chi U_{c}\left(1-U_{c}\right)\right]^{2}}{d_{1} d_{2}}\left|D^{\alpha} v(\cdot, 0)\right|^{2}\right\} \mathbf{d x} \\
+\frac{4 \widehat{C}_{1}^{2} \widehat{C}_{3}}{\lambda_{\max }} e^{2 \lambda_{\max } t}\|\mathbf{w}(\cdot, 0)\|^{2} .
\end{gathered}
$$

We will proceed in the following two cases: $\left[\chi U_{c}(1-\right.$ $\left.\left.U_{c}\right)\right]^{2} / d_{1} d_{2} \geq 1,\left[\chi U_{c}\left(1-U_{c}\right)\right]^{2} / d_{1} d_{2}<1$.

(1) If $\left[\chi U_{c}\left(1-U_{c}\right)\right]^{2} / d_{1} d_{2} \geq 1$, then it is clear from (75) that

$$
\begin{aligned}
\sum_{|\alpha|=2}\left\|D^{\alpha} \mathbf{w}(\cdot, t)\right\|^{2} \leq & \frac{\left[\chi U_{c}\left(1-U_{c}\right)\right]^{2}}{d_{1} d_{2}} \sum_{|\alpha|=2}\left\|D^{\alpha} \mathbf{w}(\cdot, 0)\right\|^{2} \\
& +\frac{4 \widehat{C}_{1}^{2} \widehat{C}_{3}}{\lambda_{\max }} e^{2 \lambda_{\max } t}\|\mathbf{w}(\cdot, 0)\|^{2} .
\end{aligned}
$$

Using (73) and (76), we know that

$$
\|\mathbf{w}(\cdot, t)\|_{H^{2}}^{2} \leq \widehat{C}_{4}\left\{\|\mathbf{w}(\cdot, 0)\|_{H^{2}}^{2}+\|\mathbf{w}(\cdot, 0)\|^{2} e^{2 \lambda_{\max } t}\right\},
$$

where $\widehat{C}_{4}=\max \left\{\left(1+C_{12}^{2}\right)\left[\chi U_{c}\left(1-U_{c}\right)\right]^{2} / d_{1} d_{2}, 4 \widehat{C}_{1}^{2}\left[1+\widehat{C}_{3}(1+\right.\right.$ $\left.\left.\left.C_{12}^{2}\right) / \lambda_{\max }\right]\right\}$.
(2) If $\left[\chi U_{c}\left(1-U_{c}\right)\right]^{2} / d_{1} d_{2}<1$, then it is not hard to verify by (70), (73), and (75) that

$$
\|\mathbf{w}(\cdot, t)\|_{H^{2}}^{2} \leq \widehat{C}_{4}\left\{\|\mathbf{w}(\cdot, 0)\|_{H^{2}}^{2}+\|\mathbf{w}(\cdot, 0)\|^{2} e^{2 \lambda_{\max } t}\right\},
$$

where $\widehat{C}_{4}=\max \left\{\left(1+C_{12}^{2}\right)\left(d_{1} d_{2} /\left[\chi U_{c}\left(1-U_{c}\right)\right]^{2}\right), 4 \widehat{C}_{1}^{2}[1+\right.$ $\left.\left.\widehat{C}_{3}\left(1+C_{12}^{2}\right) d_{1} d_{2} / \lambda_{\max }\left[\chi U_{c}\left(1-U_{c}\right)\right]^{2}\right]\right\}$ and thereby completing the proof.

\section{Main Result}

Let $\theta>0$ be a small fixed constant, and $\lambda_{\max }$ the dominant eigenvalue which is the maximal growth rate. For $\delta>0$ arbitrary small we define the escape time $T^{\delta}$ by

$$
\theta=\delta e^{\lambda_{\max } T^{\delta}}
$$

or equivalently

$$
T^{\delta}=\frac{1}{\lambda_{\max }} \ln \frac{\theta}{\delta} .
$$

Our main result in this paper reads as follows.

Theorem 6. Assume that the set of $q^{2}=\sum_{i=1}^{d} q_{i}^{2}$ satisfying instability criterion (23) is not empty for given parameters $d_{1}$, $d_{2}, \alpha, \beta, \chi, \mu$, and $U_{c}$. Let

$$
\mathbf{w}_{0}(\mathbf{x})=\sum_{\mathbf{q} \in \Omega}\left\{w_{\mathbf{q}}^{-} \mathbf{r}_{-}(\mathbf{q})+w_{\mathbf{q}}^{+} \mathbf{r}_{+}(\mathbf{q})\right\} e_{\mathbf{q}}(\mathbf{x}) \in H^{2},
$$

such that $\left\|\mathbf{w}_{0}\right\|=1$. Then there exist constants $\delta_{0}>0, \widehat{C}>0$, and $\theta>0$, depending on $d_{1}, d_{2}, \alpha, \beta, \chi, \mu$, and $U_{c}$, such that for all $0<\delta \leq \delta_{0}$, if the initial perturbation of the steady state $[\bar{U}, \bar{V}]$ is $\mathbf{w}^{\delta}(\cdot, 0)=\delta \mathbf{w}_{0}$, then its nonlinear evolution $\mathbf{w}^{\delta}(\cdot, t)$ satisfies

$$
\begin{gathered}
\left\|\mathbf{w}^{\delta}(\cdot, t)-\delta e^{\lambda_{\max } t} \sum_{\mathbf{q} \in \Omega_{\max }} w_{\mathbf{q}}^{+} \mathbf{r}_{+}(\mathbf{q}) e_{\mathbf{q}}(\mathbf{x})\right\| \\
\leq \widehat{C}\left\{e^{-\rho t}+\delta\left\|\mathbf{w}_{0}\right\|_{H^{2}}^{2}+\delta^{2}\left\|\mathbf{w}_{0}\right\|_{H^{2}}^{3}\right. \\
\left.+\delta e^{\lambda_{\max } t}+\delta^{2} e^{2 \lambda_{\max } t}\right\} \delta e^{\lambda_{\max } t}
\end{gathered}
$$

for $0 \leq t \leq T^{\delta}$, and $\rho>0$ is the gap between $\lambda_{\max }$ and the rest of $\lambda_{\mathbf{q}}$ in (82).

Proof. Let $\mathbf{w}^{\delta}(\mathbf{x}, t)$ be the solutions of (18) with initial data $\mathbf{w}^{\delta}(\cdot, 0)=\delta \mathbf{w}_{0}$. Define

$$
\begin{array}{r}
T^{*}=\sup \left\{t \mid\left\|\mathbf{w}^{\delta}(\cdot, t)-\delta e^{\mathfrak{Q} t} \mathbf{w}_{0}\right\| \leq \frac{\widehat{C}_{1}}{2} \delta e^{\lambda_{\max } t}\right\} . \\
T^{* *}=\sup \left\{t \mid\left\|\mathbf{w}^{\delta}(\cdot, t)\right\|_{H^{2}}+\left\|\mathbf{w}^{\delta}(\cdot, t)\right\|_{H^{2}}^{2}\right. \\
\left.\leq \frac{1}{\widehat{C}_{2}^{*}} \min \left\{\frac{d_{1}}{4}, \frac{\left[\chi U_{c}\left(1-U_{c}\right)\right]^{2}}{2 d_{1}}\right\}\right\} .
\end{array}
$$


We recall (80) and choose $\theta$ such that

$$
\widehat{C}_{2}^{*} \widehat{C}_{4} \theta\left(1+2 \widehat{C}_{4}^{1 / 2} \theta\right)<\min \left\{\frac{\lambda_{\max }}{4}, \frac{d_{1}}{8}, \frac{\left[\chi U_{c}\left(1-U_{c}\right)\right]^{2}}{4 d_{1}}\right\} .
$$

We first estimate $H^{2}$ norm of $\mathbf{w}^{\delta}(\mathbf{x}, t)$ for $0 \leq t \leq \min \left\{T^{\delta}\right.$, $\left.T^{*}, T^{* *}\right\}$. By the definition of $T^{*}$ and Lemma 1 , for $0<t \leq T^{*}$, we have

$$
\left\|\mathbf{w}^{\delta}(\cdot, t)\right\| \leq \frac{3}{2} \widehat{C}_{1} \delta e^{\lambda_{\max } t} .
$$

From Lemma 5, direct computation gives

$$
\left\|\mathbf{w}^{\delta}(\cdot, t)\right\|_{H^{2}} \leq \sqrt{\widehat{C}_{4}}\left\{\delta\left\|\mathbf{w}_{0}\right\|_{H^{2}}+\delta e^{\lambda_{\max } t}\right\} .
$$

It follows from (86) and $(a+b)^{p} \leq 2^{p-1}\left(a^{p}+b^{p}\right)(a, b \geq 0, p \geq$ 1) that

$$
\left\|\mathbf{w}^{\delta}(\cdot, t)\right\|_{H^{2}}^{3} \leq 4\left(\widehat{C}_{4}\right)^{3 / 2}\left\{\delta^{3}\left\|\mathbf{w}_{0}\right\|_{H^{2}}^{3}+\delta^{3} e^{3 \lambda_{\max } t}\right\} .
$$

Secondly, we establish a sharper $L^{2}$ estimate for $\mathbf{w}^{\delta}(\mathbf{x}, t)$ for $0 \leq t \leq \min \left\{T^{\delta}, T^{*}, T^{* *}\right\}$. Applying Duhamel's principle, we get

$$
\begin{aligned}
\mathbf{w}^{\delta}(\cdot, t)=\delta e^{\mathfrak{Q} t} \mathbf{w}_{0} & \\
-\int_{0}^{t} e^{\mathfrak{Q}(t-\tau)}[ & \chi\left(1-2 U_{c}\right) \nabla u^{\delta}(\tau) \nabla v^{\delta}(\tau) \\
& -2 \chi u^{\delta}(\tau) \nabla u^{\delta}(\tau) \nabla v^{\delta}(\tau) \\
& +\chi\left(1-2 U_{c}\right) u^{\delta}(\tau) \nabla^{2} v^{\delta}(\tau) \\
& -\chi\left(u^{\delta}(\tau)\right)^{2} \nabla^{2} v^{\delta}(\tau) \\
& \left.+\frac{\mu}{U_{c}}\left(u^{\delta}(\tau)\right)^{2}, 0\right] d \tau .
\end{aligned}
$$

By Lemma 1, (57), (59), and Lemma 5, for $0 \leq t \leq \min \left\{T^{\delta}\right.$, $\left.T^{*}, T^{* *}\right\}$, we know

$$
\begin{aligned}
& \left\|\mathbf{w}^{\delta}(\cdot, t)-\delta e^{\mathfrak{I} t} \mathbf{w}_{0}\right\| \\
& \quad \leq \widehat{C}_{1} \widehat{C}_{5} \int_{0}^{t} e^{\lambda_{\max }(t-\tau)}\left(\left\|\mathbf{w}^{\delta}(\tau)\right\|_{H^{2}}^{2}+\left\|\mathbf{w}^{\delta}(\tau)\right\|_{H^{2}}^{3}\right) d \tau,
\end{aligned}
$$

where $\widehat{C}_{5}=\chi\left|1-2 U_{c}\right|\left(C_{12}^{2} / C_{9}^{2}+C_{6}\right)+\mu C_{7} / \chi\left|1-2 U_{c}\right| U_{c}+$ $\chi C_{6}\left(2 C_{12}^{2} / C_{9}^{2}+1\right)$. By our choice of $t \leq \min \left\{T^{\delta}, T^{*}, T^{* *}\right\}$, it is further bounded by

$$
\begin{aligned}
&\left\|\mathbf{w}^{\delta}(t)-\delta e^{\mathfrak{l} t} \mathbf{w}_{0}\right\| \\
& \leq \widehat{C}_{1} \widehat{C}_{4} \widehat{C}_{5}\left\{\frac{\delta\left\|\mathbf{w}_{0}\right\|_{H^{2}}^{2}+4 \sqrt{\widehat{C}_{4}} \delta^{2}\left\|\mathbf{w}_{0}\right\|_{H^{2}}^{3}}{\lambda_{\max }}\right. \\
&\left.+\frac{\delta e^{\lambda_{\max } t}+2 \sqrt{\widehat{C}_{4}} \delta^{2} e^{2 \lambda_{\max } t}}{\lambda_{\max }}\right\} \delta e^{\lambda_{\max } t} .
\end{aligned}
$$

Next, We prove by contradiction that for $\delta$ sufficiently small, $T^{\delta}=\min \left\{T^{\delta}, T^{*}, T^{* *}\right\}$. If $T^{* *}$ is the smallest, we can let $t=T^{* *} \leq T^{\delta}$ in (86) and (87) to get

$$
\begin{aligned}
\left\|\mathbf{w}^{\delta}\left(T^{* *}\right)\right\|_{H^{2}}+\left\|\mathbf{w}^{\delta}\left(T^{* *}\right)\right\|_{H^{2}}^{2} \\
\leq \sqrt{\widehat{C}_{4}} \delta\left\|\mathbf{w}_{0}\right\|_{H^{2}}+\widehat{C}_{4} \delta^{2}\left\|\mathbf{w}_{0}\right\|_{H^{2}}^{2} \\
\quad+\sqrt{\widehat{C}_{4}} \theta\left(1+\sqrt{\widehat{C}_{4}} \theta\right) \\
<\frac{1}{\widehat{C}_{2}^{*}} \min \left\{\frac{d}{4}, \frac{\left[\chi U_{c}\left(1-U_{c}\right)\right]^{2}}{2 d_{1}}\right\},
\end{aligned}
$$

where $\theta$ satisfies (84) with $\widehat{C}_{4} \geq 1$ and $\delta$ is sufficiently small such that $\sqrt{\widehat{C}_{4}} \delta\left\|\mathbf{w}_{0}\right\|_{H^{2}}+\widehat{C}_{4} \delta^{2}\left\|\mathbf{w}_{0}\right\|_{H^{2}}^{2} \leq\left(1 / 2 \widehat{C}_{2}^{*}\right) \min \left\{d_{1} / 4\right.$, $\left.\left[\chi U_{c}\left(1-U_{c}\right)\right]^{2} / 2 d_{1}\right\}$. This is a contradiction to the definition of $T^{* *}$.

If $T^{*}$ is the smallest, let $t=T^{*} \leq T^{\delta}$ in (88), we see that

$$
\begin{gathered}
\left\|\mathbf{w}^{\delta}\left(\cdot, T^{*}\right)-\delta e^{\mathfrak{R} T^{*}} \mathbf{w}_{0}\right\| \\
\leq \widehat{C}_{1} \widehat{C}_{4} \widehat{C}_{5}\left\{\frac{\delta\left\|\mathbf{w}_{0}\right\|_{H^{2}}^{2}+4 \sqrt{\widehat{C}_{4}} \delta^{2}\left\|\mathbf{w}_{0}\right\|_{H^{2}}^{3}}{\lambda_{\max }}\right. \\
\left.+\frac{\theta+2 \sqrt{\widehat{C}_{4}} \theta^{2}}{\lambda_{\max }}\right\} \delta e^{\lambda_{\max } T^{*}}
\end{gathered}
$$

$$
<\frac{\widehat{C}_{1}}{2} \delta e^{\lambda_{\max } T^{*}}
$$

for $\delta$ is small such that $\widehat{C}_{4} \widehat{C}_{5}\left(\left(\delta\left\|\mathbf{w}_{0}\right\|_{H^{2}}^{2}+4 \sqrt{\widehat{C}_{4}} \delta^{2}\left\|\mathbf{w}_{0}\right\|_{H^{2}}^{3}\right) /\right.$ $\left.\lambda_{\text {max }}\right)<1 / 4$, by our choice of $\theta$ in (84) and let $\widehat{C}_{5} / \widehat{C}_{2}^{*} \leq 1$. This again contradicts the definition of $T^{*}$. Hence, $T^{\delta}$ is the smallest.

Finally, we can obtain from (31) that

$$
\begin{aligned}
& \left\|\mathbf{w}^{\delta}(\cdot, t)-\delta e^{\lambda_{\max } t} \sum_{\mathbf{q} \in \Omega_{\max }} w_{\mathbf{q}}^{+} \mathbf{r}_{+}(\mathbf{q}) e_{\mathbf{q}}(\mathbf{x})\right\| \\
& \leq\left\|\mathbf{w}^{\delta}(\cdot, t)-\delta e^{\mathfrak{\Omega} t} \mathbf{w}_{0}\right\|+\left\|\delta \sum_{\mathbf{q} \in \Omega_{\max }} w_{\mathbf{q}}^{-} \mathbf{r}_{-}(\mathbf{q}) e^{\lambda_{\mathbf{q}}^{-} t} e_{\mathbf{q}}(\mathbf{x})\right\| \\
& +\left\|\sum_{\mathbf{q} \in \Omega \backslash \Omega_{\max }}\left\{w_{\mathbf{q}}^{-} \mathbf{r}_{-}(\mathbf{q}) e^{\lambda_{\mathbf{q}}^{-} t}+w_{\mathbf{q}}^{+} \mathbf{r}_{+}(\mathbf{q}) e^{\lambda_{\mathbf{q}}^{+} t}\right\} e_{\mathbf{q}}(\mathbf{x})\right\| \\
& =\left\|\mathbf{w}^{\delta}(\cdot, t)-\delta e^{\mathfrak{\Omega} t} \mathbf{w}_{0}\right\|+I_{1}+I_{2} .
\end{aligned}
$$


By (30), (37), (39), and the definition of $\rho$, we get

$$
\begin{aligned}
I_{1}^{2} & \leq \delta^{2} e^{2\left(\lambda_{\max }-\rho\right) t}\left(\frac{\pi}{2}\right)^{d} \sum_{\mathbf{q} \in \Omega_{\max }}\left|w_{\mathbf{q}}^{-}\right|^{2}\left|\mathbf{r}_{-}(\mathbf{q})\right|^{2} \\
& \leq \delta^{2} e^{2\left(\lambda_{\max }-\rho\right) t} C_{2}^{2} C_{4}^{2}\left(\frac{\pi}{2}\right)^{d} \sum_{\mathbf{q} \in \Omega_{\max }} q^{6}\left|\mathbf{w}_{q}\right|^{2} .
\end{aligned}
$$

From (25) we know that there is one (or two) $q^{2}$ satisfying $\lambda^{+}\left(q^{2}\right)=\lambda_{\max }$. If there is only one $q^{2}$ satisfying $\lambda^{+}\left(q^{2}\right)=$ $\lambda_{\max }$, we denote it by $q_{\max }^{2}$; if there are $q_{1}^{2}$ and $q_{2}^{2}$ satisfying $\lambda^{+}\left(q_{i}^{2}\right)=\lambda_{\max }(i=1,2)$, we can let $q_{\max }^{2}=\max \left\{q_{1}^{2}, q_{2}^{2}\right\}$. It follows from (30) and (94) that

$$
I_{1} \leq \widehat{C}_{6} \delta e^{\left(\lambda_{\max }-\rho\right) t},
$$

where $\widehat{C}_{6}=C_{2} C_{3} q_{\max }^{3}$. Furthermore, by (29), (30), and the definition of $\rho$, we know that

$$
I_{2} \leq \delta e^{\left(\lambda_{\max }-\rho\right) t}
$$

Substituting (90), (95), and (96) into (93), one can obtain

$$
\begin{gathered}
\left\|\mathbf{w}^{\delta}(\cdot, t)-\delta e^{\lambda_{\max } t} \sum_{\mathbf{q} \in \Omega_{\max }} w_{\mathbf{q}}^{+} \mathbf{r}_{+}(\mathbf{q}) e_{\mathbf{q}}(\mathbf{x})\right\| \\
\leq \widehat{C}\left\{e^{-\rho t}+\delta\left\|\mathbf{w}_{0}\right\|_{H^{2}}^{2}+\delta^{2}\left\|\mathbf{w}_{0}\right\|_{H^{2}}^{3}\right. \\
\left.+\delta e^{\lambda_{\max } t}+\delta^{2} e^{2 \lambda_{\max } t}\right\} \delta e^{\lambda_{\max } t}
\end{gathered}
$$

where $\widehat{C}=\max \left\{\widehat{C}_{6}+1,4 \widehat{C}_{1} \widehat{C}_{4}^{3 / 2} \widehat{C}_{5} / \lambda_{\text {max }}\right\}$ with $\widehat{C}_{4} \geq 1$ and thereby concluding the proof.

Theorem 6 implies that the dynamics of a general perturbation are characterized by such linear dynamics over a long time period of $\varepsilon T^{\delta} \leq t \leq T^{\delta}$, for any $\varepsilon>0$. In particular, choose a fixed $\mathbf{q}_{0}=\left(q_{01}, q_{02}, \ldots, q_{0 d}\right) \in \Omega_{\max }$ and let $\mathbf{w}_{0}(\mathbf{x})=$ $\kappa\left(\mathbf{r}_{+}\left(\mathbf{q}_{0}\right) /\left|\mathbf{r}_{+}\left(\mathbf{q}_{0}\right)\right|\right) e_{\mathbf{q}_{0}}(\mathbf{x})$, where $\kappa=1 /\left\|e_{\mathbf{q}_{0}}\right\|=\sqrt{(2 / \pi)^{d}}$ so that $\left\|\mathbf{w}_{\mathbf{0}}(\mathbf{x})\right\|=1$. Then

$$
\left\|\mathbf{w}_{0}(\mathbf{x})\right\|_{H^{2}}=\left(1+\left|\mathbf{q}_{0}\right|^{2}+\left|\mathbf{q}_{0}\right|^{4}\right)^{1 / 2} .
$$

Therefore, if $t \leq T^{\delta}$, we can obtain from (97) and (98)

$$
\begin{gathered}
\left\|\mathbf{w}^{\delta}\left(\cdot, T^{\delta}\right)-\delta e^{\lambda_{\max } T^{\delta}} \kappa \frac{\mathbf{r}_{+}\left(\mathbf{q}_{0}\right)}{\left|\mathbf{r}_{+}\left(\mathbf{q}_{0}\right)\right|} e_{\mathbf{q}_{0}}(\mathbf{x})\right\| \\
\leq \widehat{C}_{8}\left\{\delta^{\rho / \lambda_{\max }}+\theta^{2}+\theta^{3}\right\}
\end{gathered}
$$

where $\widehat{C}_{8}=\widehat{C} \max \left\{\theta^{1-\left(\rho / \lambda_{\max }\right)},\left(1+\left|\mathbf{q}_{0}\right|^{2}+\left|\mathbf{q}_{0}\right|^{4}\right)^{3 / 2}+1\right\}$. If $0<$ $\theta<\left(1 / \widehat{C}_{8}\right)^{3 / 2}$ and $\delta_{0}=\left(\theta / 2 \widehat{C}_{8}-\theta^{2}-\theta^{3}\right)^{\lambda_{\max } / \rho}$, then

$$
\begin{aligned}
\left\|\mathbf{w}^{\delta}\left(\cdot, T^{\delta}\right)\right\| \geq \theta-\widehat{C}_{8}\left\{\delta^{\rho / \lambda_{\max }}+\theta^{2}+\theta^{3}\right\} & \geq \frac{\theta}{2}>0, \\
0 & <\delta \leq \delta_{0} .
\end{aligned}
$$

This means nonlinear instability as $\delta \rightarrow 0$.
Respecting the above-mentioned facts, the models (7) and (13) have no nonconstant positive steady state no matter what the self-diffusion coefficients $d_{1}, d_{2}$ and cross diffusion $d_{3}$ are; in other words, self-diffusion and cross diffusion (without chemotaxis) cannot drive instability and cannot generate patterns for the corresponding model. This means that chemotaxis-driven instability occurs. We prove that for any given general perturbation of magnitude $\delta$, linear fastest growing modes determine the nonlinear evolution for the model (2), over a time period of the order $\ln 1 / \delta$. Therefore, our results indeed provide a rigorous mathematical description for the nonlinear pattern formation in a volume-filling chemotaxis model with logistic growth.

\section{Conflict of Interests}

The authors declare that they have no conflict of interests regarding the publication of this paper.

\section{Acknowledgments}

This work is supported by the China National Natural Science Foundation (nos. 11361055 and 11261053) and the Fundamental Research Funds for the Gansu University.

\section{References}

[1] K. J. Painter and T. Hillen, "Volume-filling and quorum-sensing in models for chemosensitive movement," The Canadian Applied Mathematics Quarterly, vol. 10, no. 4, pp. 501-543, 2002.

[2] T. Hillen and K. Painter, "Global existence for a parabolic chemotaxis model with prevention of overcrowding," Advances in Applied Mathematics, vol. 26, no. 4, pp. 280-301, 2001.

[3] D. Wrzosek, "Global attractor for a chemotaxis model with prevention of overcrowding," Nonlinear Analysis: Theory, Methods \& Applications, vol. 59, no. 8, pp. 1293-1310, 2004.

[4] D. Wrzosek, "Long-time behaviour of solutions to a chemotaxis model with volume-filling effect," Proceedings of the Royal Society of Edinburgh A: Mathematics, vol. 136, no. 2, pp. 431-444, 2006.

[5] A. B. Potapov and T. Hillen, "Metastability in chemotaxis models," Journal of Dynamics and Differential Equations, vol. 17, no. 2, pp. 293-330, 2005.

[6] V. Calvez and J. A. Carrillo, "Volume effects in the KellerSegel model: energy estimates preventing blow-up," Journal de Mathématiques Pures et Appliquées, vol. 86, no. 2, pp. 155-175, 2006.

[7] M. Burger, M. di Francesco, and Y. Dolak-Struss, "The KellerSegel model for chemotaxis with prevention of overcrowding: linear vs. nonlinear diffusion," SIAM Journal on Mathematical Analysis, vol. 38, no. 4, pp. 1288-1315, 2006.

[8] Z. Wang and T. Hillen, "Classical solutions and pattern formation for a volume filling chemotaxis model," Chaos, vol. 17, no. 3, Article ID 037108, 13 pages, 2007.

[9] J. Jiang and Y. Zhang, "On convergence to equilibria for a chemotaxis model with volume-filling effect," Asymptotic Analysis, vol. 65, no. 1-2, pp. 79-102, 2009.

[10] D. Wrzosek, "Volume filling effect in modelling chemotaxis," Mathematical Modelling of Natural Phenomena, vol. 5, no. 1, pp. 123-147, 2010. 
[11] M. Winkler, "Does a "volume-filling effect" always prevent chemotactic collapse?" Mathematical Methods in the Applied Sciences, vol. 33, no. 1, pp. 12-24, 2010.

[12] M. Winkler and K. C. Djie, "Boundedness and finite-time collapse in a chemotaxis system with volume-filling effect," Nonlinear Analysis: Theory, Methods \& Applications, vol. 72, no. 2, pp. 1044-1064, 2010.

[13] Z. Wang, M. Winkler, and D. Wrzosek, "Singularity formation in chemotaxis systems with volume-filling effect," Nonlinearity, vol. 24, no. 12, Article ID 3279, 2011.

[14] Y. Y. Zhang and S. M. Zheng, "Asymptotic behavior of solutions to a quasilinear nonuniform parabolic system modelling chemotaxis," Journal of Differential Equations, vol. 248, no. 7, pp. 1684-1710, 2010.

[15] Y. Zhang and S. Zheng, "A 1-d quasilinear nonuniform parabolic chemotaxis model with volume-filling effect," Asymptotic Analysis, vol. 83, no. 1-2, pp. 101-125, 2013.

[16] F. Li and Y. Y. Zhang, "On steady-state solutions of a 1-D chemotaxis model with volume-filling effect," Journal of Mathematical Analysis and Applications, vol. 408, no. 1, pp. 1-18, 2013.

[17] J. I. Tello and M. Winkler, "A chemotaxis system with logistic source," Communications in Partial Differential Equations, vol. 32, no. 4-6, pp. 849-877, 2007.

[18] M. Winkler, "Boundedness in the higher-dimensional parabolic-parabolic chemotaxis system with logistic source," Communications in Partial Differential Equations, vol. 35, no. 8, pp. 1516-1537, 2010.

[19] K. J. Painter and T. Hillen, "Spatio-temporal chaos in a chemotaxis model," Physica D: Nonlinear Phenomena, vol. 240, no. 4-5, pp. 363-375, 2011.

[20] S. Banerjee, A. P. Misra, and L. Rondoni, "Spatiotemporal evolution in a $(2+1)$-dimensional chemotaxis model," Physica A: Statistical Mechanics and Its Applications, vol. 391, no. 1-2, pp. 107-112, 2012.

[21] K. Kuto, K. Osaki, and T. a. . Sakurai, "Spatial pattern formation in a chemotaxis-diffusion-growth model," Physica D: Nonlinear Phenomena, vol. 241, no. 19, pp. 1629-1639, 2012.

[22] S. Fu and J. Liu, "Spatial pattern formation in the Keller-Segel model with a logistic source," Computers \& Mathematics with Applications, vol. 66, no. 3, pp. 403-417, 2013.

[23] C. Ou and W. Yuan, "Traveling wavefronts in a volume-filling chemotaxis model," SIAM Journal on Applied Dynamical Systems, vol. 8, no. 1, pp. 390-416, 2009.

[24] M. Ma, C. Ou, and Z. Wang, "Stationary solutions of a volume-filling chemotaxis model with logistic growth and their stability," SIAM Journal on Applied Mathematics, vol. 72, no. 3, pp. 740-766, 2012.

[25] M. Ma, J. Hu, J. Tao, and C. Tong, "Non-existence of stationary pattern of a chemotaxis model with logistic growth," Nonlinear Analysis: Theory, Methods \& Applications, vol. 105, no. 1, pp. 3-9, 2014.

[26] Y. Guo and H. J. Hwang, "Pattern formation (I): the Keller-Segel model," Journal of Differential Equations, vol. 249, no. 7, pp. 15191530, 2010.

[27] S. M. Fu and J. Liu, "A mathematical characterization for patterns of a Keller-Segel model with a cubic source term," Advances in Mathematical Physics, vol. 2013, Article ID 934745, 11 pages, 2013.

[28] Y. Guo and W. A. Strauss, "Instability of periodic BGK equilibria," Communications on Pure and Applied Mathematics, vol. 48, no. 8, pp. 861-894, 1995.
[29] J. K. Hale, Ordinary Differential Equations, Krieger, Malabar, Fla, USA, 1980.

[30] D. Henry, Geometric Theory of Semilinear Parabolic Equations, vol. 840 of Lecture Notes in Mathematics, Springer, New York, NY, USA, 1993.

[31] O. A. Ladyzenskaja, V. A. Solonnikov, and N.N. Uralceva, Linear and Quasilinear Equations of Parabolic Type, Translations of Mathematical Monographs, American Mathematical Society, Providence, RI, USA, 1968.

[32] M.X. Wang, Nonlinear Partial Differential Equations of Parabolic Type, Science Press, Beijing, China, 1993. 


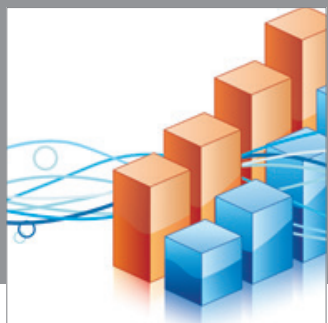

Advances in

Operations Research

mansans

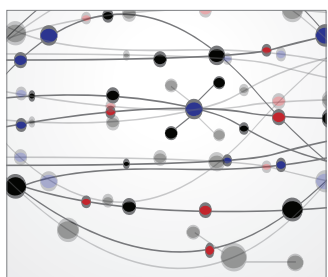

The Scientific World Journal
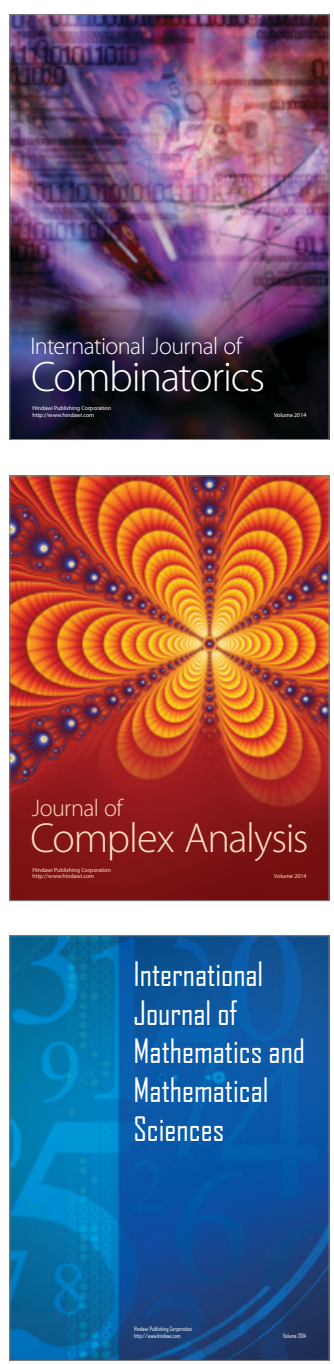
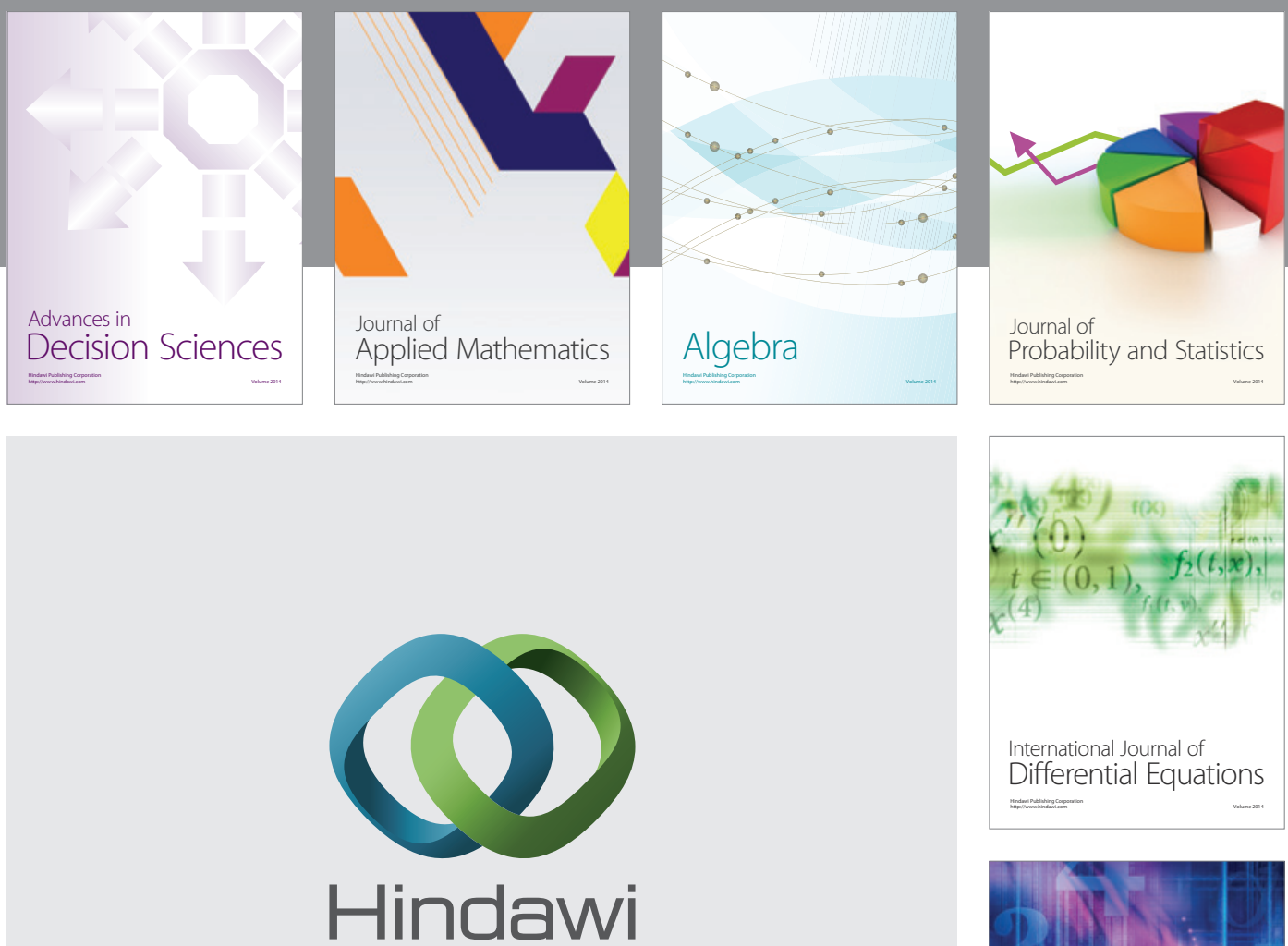

Submit your manuscripts at http://www.hindawi.com
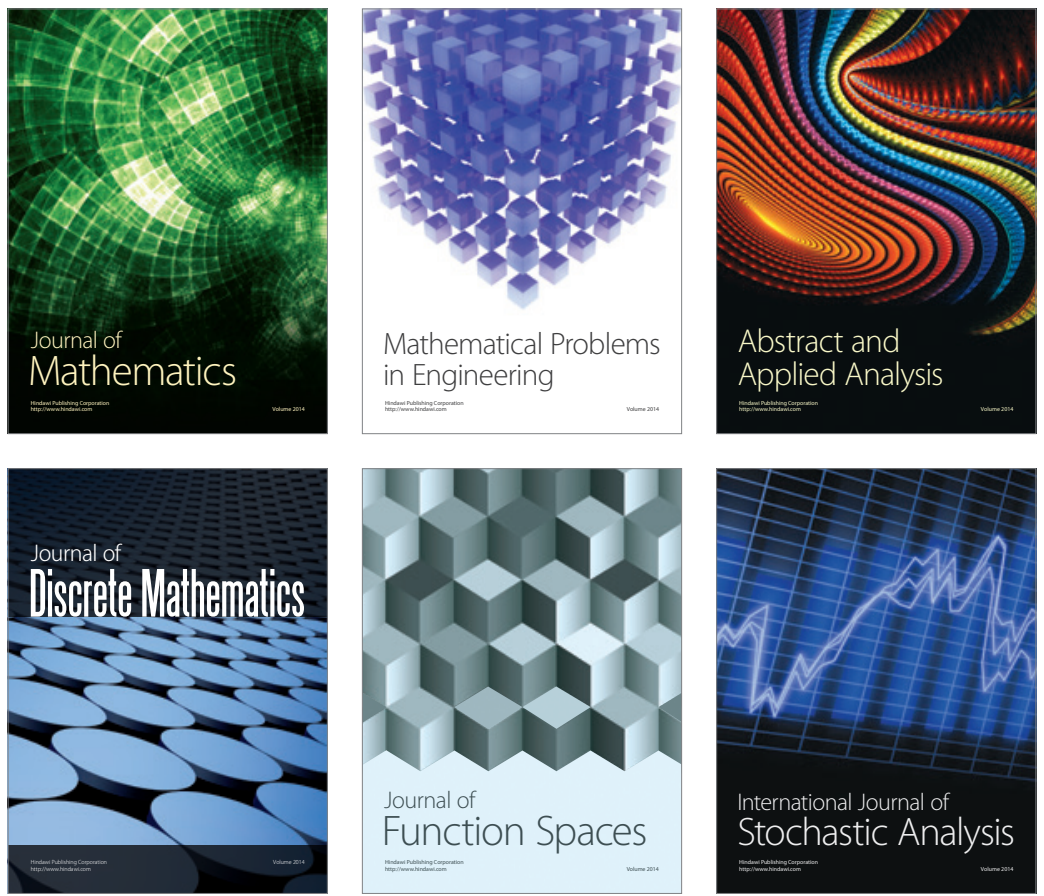

Journal of

Function Spaces

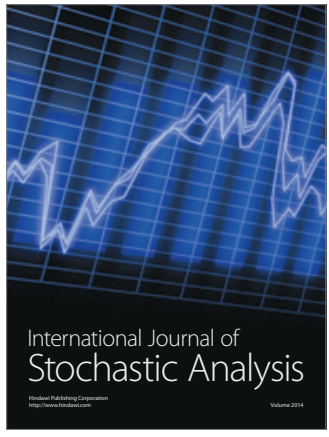

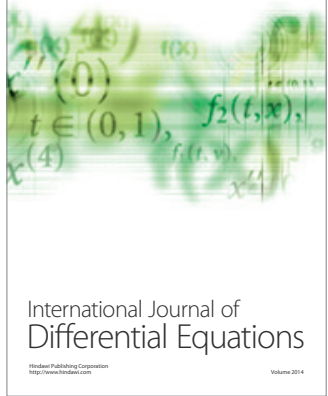
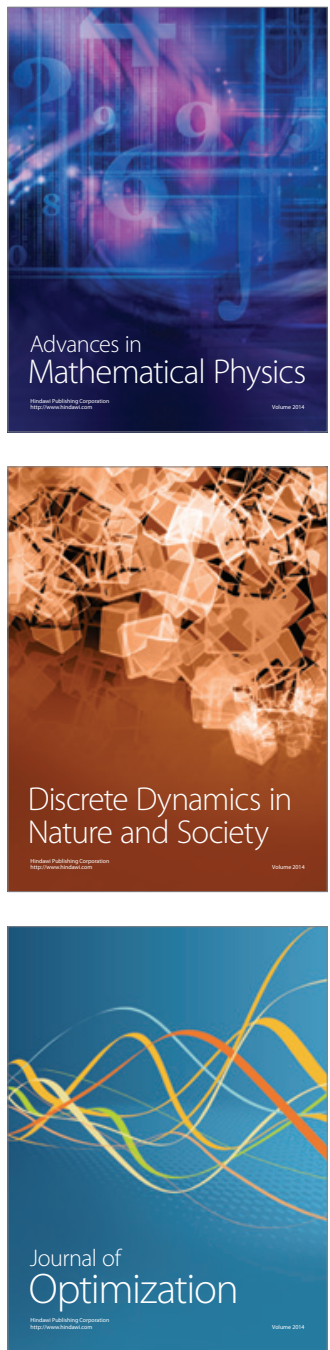